\title{
GPS 技术在水利工程测量中的应用
}

\author{
李嘉智 \\ 辽宁省阜新市水利事物服务中心 \\ DOI:10.32629/hwr.v3i7.2297
}

[摘 要] 工程测量在水利工程中发挥着重要作用,其能够有效保证水利工程的质量。随着科学技术的进步,近年来先进的工程 测量技术逐渐被开发并应用到水利工程中。本文主要针对 GPS 技术工作原理及组成和特点、GPS 技术在水利工程测量中的 应用、GPS 技术在水利工程测量中的不足以及相应的对策做了具体分析,以供参考。

[关键词] GPS 技术; 水利工程; 工程测量; 应用

\section{引言}

水利工程不仅可以给人类生活和生产带来巨大的便利, 还能够有效促进我国经济的发展和社会的进步, 要想保证水 利工程的质量, 就需要做好工程测量工作。在科学技术不断 进步的背景下, GPS测量技术的应用使得水利工程测量工作 更加便捷, 测量精度也更高。但是就目前来看, GPS测量技术 仍旧有一些不足, 有待进一步改变。

\section{GPS 技术工作原理、组成及其特点}

1. 1 GPS技术工作原理

对于GPS技术来说, 其主要就是应用距离交会法来完成 对工程的测量。就目前来看, 工程中常用的GPS坐标系统有两 个, 一个是地固坐标系统, 另一个是空间固定坐标系统, 这两 种系统没有固定的界限, 在使用时可以进行有效转换, 从而 更好地控制测量点的坐标。

\subsection{GPS组成}

具体来说, GPS系统主要由三部分组成, 分别是空间卫星 群、地面监控系统和卫星接收设备。首先来看空间卫星群, 之所以被称为卫星群, 是因为其不是由一颗卫星工作, 而是 分布在 6 个轨道上的 24 颗GPS卫星共同工作, 这些GPS卫星运 行轨道和地球赤道的夹角大概是 $55^{\circ}$, 只有这样才能接收到 更多的信号, 最多时可以接收到11颗GPS卫星发射的信号。再 来看地面监控系统, 该系统也比较复杂, 不仅需要建设主控 站来计算卫星的星历和卫星钟的修改参数, 还需要建立 5 个 监测站用以接收GPS卫星发射的信号, 另外, 还需要建立相应 的注入站, 通常是 2 个, 注入站的主要功能也是接收功能, 只 不过其接收的是修改参数, 这些组成各自发挥着各自的作用, 又相互影响, 相互联系, 促进测量工作的顺利开展。最后来看 卫星接收设备, 这些设备位于用户手中, 接收机通过对接收 到的信号分析能够有效完成导航与定位工作。

\subsection{GPS技术的特点}

GPS技术在水利工程测量中目前已经处于领先地位, 这 主要是因为它的功能与优点非常多, 并且较为突出。与传统 的测量技术相比, 其不仅可以有效提高测量精度, 缩短测量 时间, 还能够为测量人员提供三维坐标 ${ }^{[1]}$, 进行全天候作业, 并且使用该技术进行测量时能不需要繁琐的操作步骤, 使用
便捷。GPS技术测量精度高具体表现为: 如果工程测量精密 定位是 $300 \mathrm{~m}-1500 \mathrm{~m}$, 那么使用 GPS技术测量出来的平面位置 误差最大为 $1 \mathrm{~mm}$, 这为提高水利工程质量提供了有力保障。除 此之外, GPS技术在工程测量中不仅可以进行位置测量, 还 能够进行测速与测时, 应用范围非常广。相信在未来的发展 中, GPS技术会更加成熟, 其对水利工程测量的作用将更加 明显。

\section{GPS 技术在水利工程测量中的应用}

2. 1 外业测量

在水利工程测量中, 要保证测量结果是准确的、可靠的, 就需要保证测量点的选取是合理的、有代表性的, 而GPS技术 恰好可以对测量范围内的地理信息进行全面获取, 因此, 相 关人员可以利用GPS技术进行测量, 通过对测量范围内的地 理数据进行科学分析来找出最佳测量点。在使用GPS技术时, 需要在测量现场安装无线, 为了保证在大风等天气时仍能正 常测量, 需要对架设的天线进行固定。

\section{2 布网测量}

在水利工程测量中, 布网工作非常重要, 不同的工程项 目对应不同的布网方式, 因此, 在进行选择时应对工程要求 进行合理分析, 例如, 在引水工程此类带状工程的测量中, 为 提高测量效率和质量, 最常用的方式是点连式布网; 而对于 工程枢纽区域的施工控制网及变形监测网, 为进一步加强网 形的集合强度, 并确保GPS控制网的可靠性以及数据的准确 性, 通常采用边连式或网连式进行布设 ${ }^{[2]}$ 。

\section{3 堤防工程施工测量}

对于堤防工程施工测量来说, 如果使用传统的测量方式, 只能对平面和高程采取分级布置的方式, 这种方式在测量时 很难保证测量精度, 也很难保证分层竞速的均匀性, 测量结 果误差明显。此外, 由于观测测回数目较多, 观测的精确度 有待提高, 同时受环境影响较大, 高程测量往往费时费力。 而GPS技术则可以有效进行动态测量, 不必要进行平面控制 点的累计传递, 由此一来就可以提高测量精度, 保证测量的 质量。

2.4大型水工建筑物变形观测

GPS技术在大型水工建筑物的变形测量中同样有着重要 
的应用。在使用GPS技术对大型水工建筑物的变形情况进行 监测时, 只需要安装好监测仪器就行, 不必设定基准点。倘若 观测点无法顺利进行设置, 那么应用GPS测量就可以对基准 点的稳定性进行快速方便的核定, 并不需要布置参考网格, 在很大程度上保证了工作效率。

\section{GPS 技术在水利工程测量中的不足}

3.1 GPS技术在测量中存在误差

虽然GPS技术的优势很多, 但是在实际应用过程中, 其仍 旧存在一定的测量误差, 这主要体现在高山和森林等地势条 件恶劣或者建筑物过于密集的地区的测量, 在这些地区进行 测量时由于GPS卫星信号会受到遮挡而影响传输效率, 从而 影响测量精度。还有一些操作人员因为一时的疏忽, 或观测 的角度不对等原因也会导致GPS技术在水利工程测量中存在 着一些误差。

\section{2 操作人员不熟练}

有些施工测量单位为了获取较高的经济效益而雇佣专 业水平不高的人员, 这些人员在测量作业中不能熟练应用 GPS技术, 缺乏对GPS技术原理及相关知识的掌握, 从而影响 测量结果。

\section{3 应用力度有待提高}

水利工程测量中采用的电子全站仪和电子水准仪等先 进的设备 ${ }^{[3]}$, 在技术要求上已经很成熟, 基本能满足测量的 需要。但是因为GPS技术应用时间并不长, 因此在应用力度上 还有很大的进步空间。

\section{4 提高 GPS 技术的措施}

4. 1加强培训, 提高技术人员的素质

第一, 对GPS技术的工作原理、机器构造、具体操作进行 详细讲解, 使技术人员对GPS技术熟练操作; 第二, 由于水利
工程测量都是户外作业, 有些项目的作业环境较为艰苦, 因 此, 应当对技术人员的心理素质进行培训, 提高其适应艰苦 环境的能力, 当遇到突发情况时, 其能够自如应对, 从而降低 差错率。

4. 2 加强宣传, 提高GPS技术在水利工程中的应用

GPS技术是一种精度比较高的测量技术, 采用的是全球 定位系统, 提高了测量的精确度和效率。对此, 相关人员应进 一步加强对GPS技术的宣传, 让更多的人了解GPS技术的优势, 从而扩大其应用空间。

4. 3 构建GPS与其他变形监测技术组合成的综合变形监 测系统

虽然GPS技术在变形监测中的应用有较强的优势, 但是 也存在一定的不足, 而通过与其他变形监控技术的紧密结 合应用, 可以实现不同监测技术间的优势互补, 由此一来就 可以将GPS的优势最大化，从而为水利工程测量提供更好的 服务。

\section{5 结语}

综上所述, 随着科学技术的不断发展, GPS技术逐渐得到 完善, 将其应用到水利工程中能够有效提高测量精度与测量 效率, 保证测量质量, 在很大程度上推动了水利工程的进一 步发展。

[参考文献]

[1]徐立军.GPS技术在水利测量工程中的应用 [J]. 黑龙 江水利科技,2017(03):135-137.

[2]倪南钊.关于GPS技术在水利工程测量中的应用分析 [J].科技风,2017(07):214.

[3]鞠海明,陈启涛.GPS技术在水利工程测量中的应用 [J]。 电子测试,2018(19):62-63. 